\title{
WATER AND ELECTROLYTE BALANCE IN DISEASE
}

\author{
By James Conway, M.B., J. Lee, M.B., M.R.C.P., and W. O. SyKes, M.Sc. \\ Charing Cross Hospital Medical School
}

Many medical and surgical emergencies are complicated by derangement of water and electrolyte balance, the successful management of which depends upon the rapid assessment of essential clinical and laboratory data, and the prompt institution of a certain pre-arranged routine of treatment. An attempt is made in this review to illustrate the application of physiological principles to specific clinical problems.

The major part $(351$.$) of the water content of$ the body is an integral part of the cell structure, the remainder ( 151 .) is extracellular fluid comprising intersitial fluid and plasma. The composition of the fluid in the two compartments is different. Potassium and phosphate are the main cation and anion respectively of the cells, while sodium and chloride preponderate in the extracellular fluid. The difference in ionic composition between the two compartments is well maintained in health, the distribution of water between the cells and the fluid bathing them being regulated by the osmotic pressure of solutes. An increased concentration of substances on one side of the membrane will draw water from the other.

The extracellular fluid acts as a buffer between the cells and the external environment with which it is in constant contact through the lungs, skin, gut and kidney. It suffers greater proportional changes in volume than the cellular fluid.

The concentration of solutes in body fluids is regulated by receptors in the hypothalamus sensitive to certain changes in the osmotic pressure of the plasma. The reabsorption of water by the renal tubules is then controlled by the production of the antidiuretic hormone of the posterior pituitary. ${ }^{1}$

The mechanism whereby the volume of body fluids, particularly the extracellular fluid, is regulated, is not known. A mechanism analogous to that controlling osmotic pressure has been postulated, ${ }^{2}$ whereby a receptor sensitive to changes in blood volume or flow stimulates the production of a hormone, possibly of the adrenal cortex, which, by adjusting the amount of salt reabsorbed, will regulate blood volume and indirectly extracellular fluid volume.

Disturbances of water and electrolyte balance will be considered under two headings: (a) Water and salt balance; (b) potassium balance.

\section{Water and Salt Balance}

Disturbances of electrolyte balance affect $\vec{D}$ primarily the volume of the extracellular fluids, while excess or loss of water will be distributed uniformly throughout the body. When the diss turbances persist, or become more severe, cellulad function is affected and further changes are, i $\vec{G}$ consequence, superimposed on pre-existing abnote malities.

Marriott ${ }^{3}$ stressed the importance of evaluating separately losses of water and salt, and on Table $I$ the signs and symptoms of pure water and pure salt are presented.

Losses of water and salt do not occur independently, but against the background of this separation of the two distinct processes, deviations from the normal fluid and electrolyte balance in disease may be divided artificially into five clinical states: (I) Primary loss of sodium; (2) primary excess of sodium; (3) primary excess of saline; (4) primary retention of water; and (5) primary loss of water.

\section{Primary Loss of Sodium}

Loss of electrolyte from the extracellular fluid involves chiefly sodium and chloride ions. Retention of $\mathrm{CO}_{2}$ allows easy replacement of lost anions $(\mathrm{Cl}-)$ by bicarbonate ions $\left(\mathrm{HCO}_{3}-\right)$ without ill effect. The cations $\left(\mathrm{Na}^{+}\right)$however can only be replaced from the exterior, therefore only a small loss can be tolerated. Loss of sodium will tend to reduce the osmotic pressure of the extracellular fluid, with the result that water will pass into the cells and the kidneys will excrete a dilute urine. Thus the first compensatory mechanism is to maintain the osmotic pressure of body fluids at the 
expense of extracellular fluid volume. The plasma, being one-third of this fluid, will suffer a simultaneous reduction in volume.

The degree of deficiency of sodium cannot be gauged with accuracy from the plasma sodium, for the essential feature of this condition is the reduction of extracellular volume, which may be measured indirectly by the reduction in plasma volume. This diminution in circulatory fluid is reflected in an increase in packed cell volume and concentration of plasma proteins. A full and vivid description of the effects of sodium deficiency is given by McCance in the Gouldstonian Lectures of 1936.4

Sodium deficiency occurs classically in Addison's disease where the ability of the renal tubules to reabsorb sodium is impaired and even in the presence of a deficiency the kidney continues to excrete sodium.

When considerable quantities of fluid are lost from the gut in persistent vomiting, intestinal suction, pancreatic or biliary fistulae and severe diarrhoea, the loss of sodium becomes important. Excessive sweating will also carry away large quantities of sodium. When allowed free access to water these cases will develop sodium deficiency.

The peripheral circulatory failure which is an essential feature of diabetic acidosis results from the continued loss of sodium in the urine. In renal disease, when tubular exceeds glomerular damage, the ability of the kidneys to conserve sodium is impaired, and a condition clinically similar to Addison's disease may appear. These patients therefore cannot withstand a dietary deficiency of sodium, and treatment of hypertension with a low sodium diet may thus be dangerous.

\section{Primary Retention of Sodium}

This state, the reverse of that just described, must lead by the retention of water to the expansion of extracellular fluid volume at the expense of ingested water and intracellular fluid. The condition arises when hypertonic sodium solutions are ingested and access to water is restricted. This will occur on the ingestion of sea water by the shipwrecked, or after infusions of normal saline (.9 per cent.). The fluid balance of a patient whose requirements are met solely by the administration of normal saline may be given as follows:-

Daily fluid requirement, 3 pt. or $1,500 \mathrm{cc}$ of saline.

$$
\begin{aligned}
& \text { Intake } \\
& \begin{array}{llllll}
\text { Water } & \ldots & \ldots & \ldots & \mathbf{1}, 500 & \text { cc. }
\end{array} \\
& \begin{array}{llllll}
\mathrm{NaCl} & \ldots & \ldots & \ldots & \ldots & \text { I3.5 gm. }
\end{array} \\
& \text { Loss } \\
& \begin{array}{llll}
\text { Lungs } & \ldots & \ldots & 600 \mathrm{cc} \text {. I Inevitable } \\
\text { Skin } & \ldots & \ldots & 400 \mathrm{cc} . \text { S }^{2}
\end{array} \\
& \text { Total .. r,000 cc. Lost as pure }
\end{aligned}
$$

Thus $13.5 \mathrm{gm}$. of $\mathrm{NaCl}$ remains to be excreted by the kidneys with $500 \mathrm{cc}$. water, i.e. 2.7 per cent. $\mathrm{NaCl}$. The kidneys are unable to accomplish this, and the resulting sodium retention will draw watero from the cells causing intracellular dehydration.

The administration of larger quantities of saline to cover inevitable losses and renal requirements has its own danger. When isotonic saline is given it does not stimulate a prompt diuresis as does water ; it is excreted over a period of days. Much of the saline is retained expanding the extracellular fluid and giving rise to dangers of heart failure and pulmonary oedema.

TABLE I

Comparison of Effects of Water and Salt Depletion

\begin{tabular}{l|l|l}
\multicolumn{1}{c|}{ Manifestation } & \multicolumn{1}{c}{ Pure Water Depletion } & \multicolumn{1}{c}{ Pure Salt Depletion } \\
\hline $\begin{array}{l}\text { Dehydration } \\
\text { Thirst }\end{array}$ & +++ primary or simple & +++ secondary or extracellular \\
Lassitude & +++ & Absent \\
Orthostatic fainting & Absent till late & ++ \\
Urine volume & Scanty & Normal till late \\
NaCl in urine & Often + & Always absent except in Addison's disease \\
Vomiting & Absent & May be +++ \\
Cramps & Absent & May be +++ \\
Plasma NaCl & Slight increase or normal & + \\
Blood urea & + & ++ \\
Haemoconcentration & Not till late and slight & Increased +++ \\
Blood viscosity & Normal till late & Fall +++ \\
Blood pressure & Normal till late & Slow \\
Water absorption & Rapid & Peripheral circulatory failure \\
Mode of death & ? due to rise of osmotic pressure & .
\end{tabular}

Reproduced by kind permission of Dr. H. L. Marriott and the Editor of the British Medical Fournal 


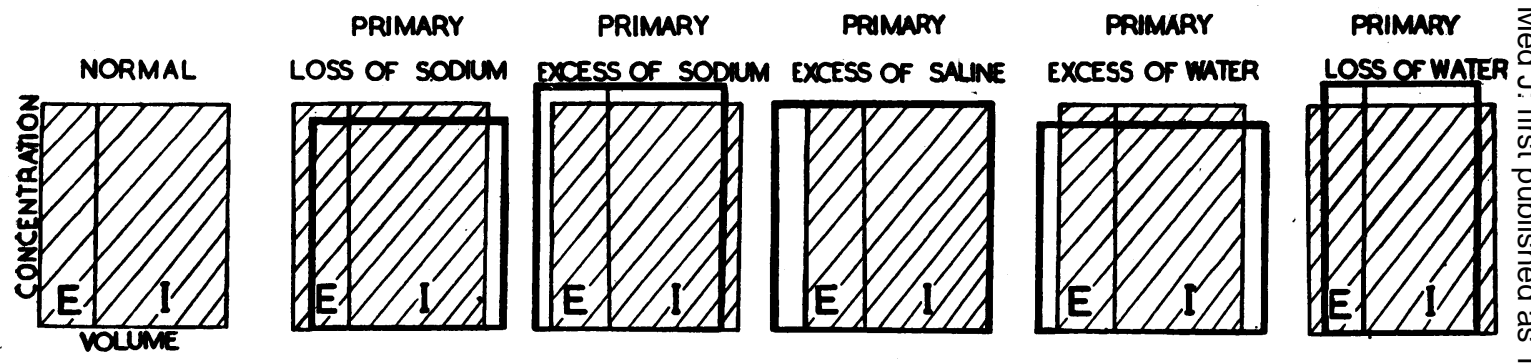

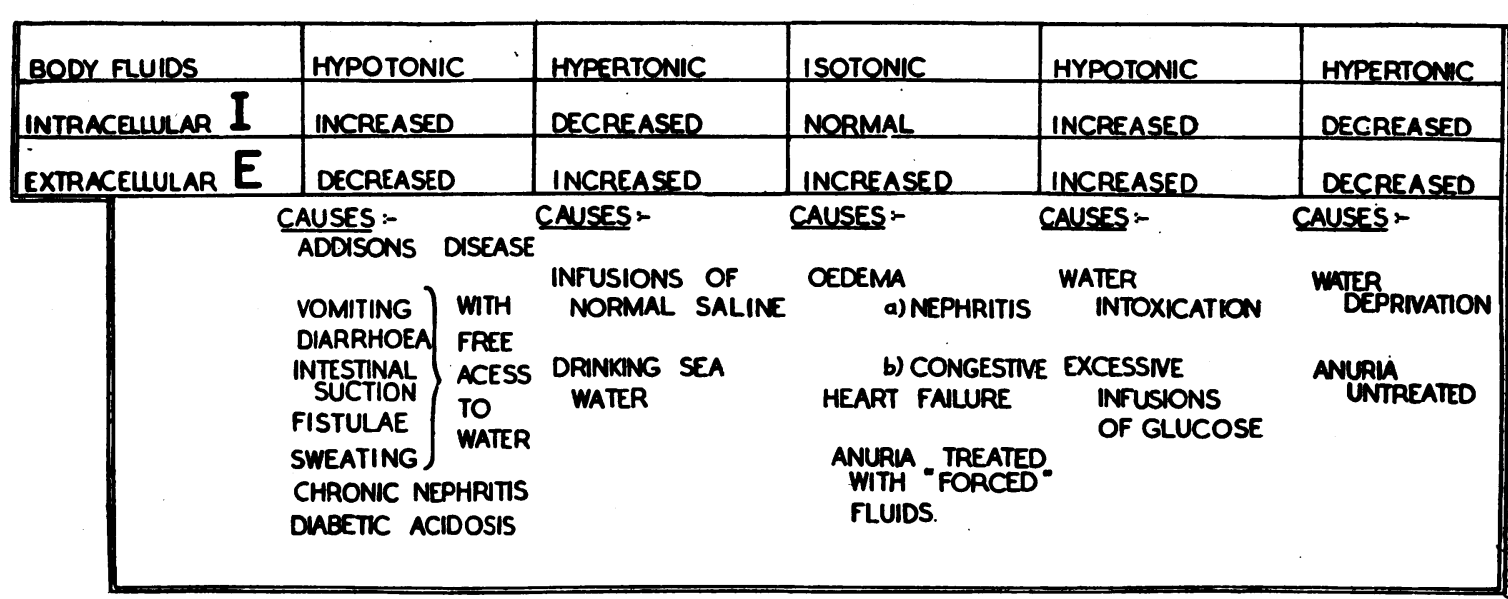

Normal saline therefore should never be the sole source of fluid to any patient unless there is a demonstrable deficiency of sodium. This applies particularly to patients in the first week after operation in whom the ability to excrete sodium chloride has been shown to be greatly reduced. ${ }^{5}$ If it is necessary after an operation to maintain the patient by intravenous fluids, the salt content should be strictly related to the actual daily loss by the kidneys.

\section{Primary Retention of Isotonic Fluid}

The retention of isotonic fluid must be regarded as the cause of oedema and therefore separated from primary retention of either sodium or water. In Type 2 nephritis the continued loss of plasma protein leads to retention of salt and water in the body. The cause of oedema in congestive heart failure is difficult to explain. Neither the rise in venous pressure nor the increased permeability of capillary membranes due to anoxia can explain the presence of the oedema. Studies of renal function in heart failure show that there is a reduction in the renal blood flow and in the glomerular filtration rate; the effect of these is to increase abnormally the reabsorption of sodium by the tubules. ${ }^{6,7}$

It is important to realize that sodium is retained with water in the oedema; patients are 'brine- logged ' and not waterlogged. ${ }^{8}$ The success of the rigid restriction of sodium ( $0.5 \mathrm{gm}$./day) in heart failure thus becomes apparent. Water restriction in oedema will produce an increase in the concentration of sodium in extracellular fluid and a withdrawal of water from the cells. This cellular dehydration is responsible for the paradox of severe and distressing thirst existing in a grossly oedematous patient. The efficiency of mercurial diuretics in heart failure has been shown to be due to their ability to increase the excretion of sodium. ${ }^{9}$ Ion-exchange resins are being developed which, by their ability to absorb sodium ions, can prevent their absorption from the gut even after the normal amount is taken in the diet. ${ }^{10}$

\section{Primary Retention of Water}

In normal circumstances the kidneys are capable of rapid elimination of large quantities of ingested water, and the danger of water intoxication appears only as a result of artificial or diseased states which prevent the normal prompt diuresis. In practice this state occurs when renal function has been grossly depressed. As water accumulates within the body it will be distributed throughout all fluid compartments, increasing their volume but decreasing their osmotic pressure. The signs and symptoms of an excess of water appear to be due 
to changes within the cells. There is restlessness, delirium, coma, irregular muscle twitching, cramps and sometimes convulsions. If water requirement is over estimated and excessive infusions of isotonic glucose given, or liberal amounts are taken orally, a condition analagous to water intoxication may occur.

\section{Primary Loss of Water}

Loss of water from the body will lead to a progressive increase in the osmotic pressure of all compartments of body fluid. The cellular loss of fluid is an important cause of the changes in potassium balance which will be considered in the next section. 'Throughout a period of deprivation the inevitable loss daily of about 1,000 cc. of pure water will cor tinue, aggravating the water deficit.

The kidneys, in an attempt to conserve water, will concentrate the urine maximally to a specific gravity of 1032 , and will pass a steady minimal amount of urine-about $500 \mathrm{cc}$ - - until renal blood flow, and hence the glomerular filtrate, is reduced, with ensuing pre-renal uraemia.

To reduce the body requirement of water two principles of management apply - to eliminate the intake of electrolyte and to reduce the production of urea to a minimum by giving a diet of adequate calorific value but no protein.

These principles are directly applicable to the treatment of cases of anuria. A programme of treatment must be prepared and be ready for use whenever a case of anuria appears; it may be modelled on those of Muirhead ${ }^{11}$ and Bull. ${ }^{12}$

It is important to realize that when the kidneys are producing no urine the body is unable to lose electrolyte. It is essential therefore that no electrolytes whatsoever should be given. The water required to cover daily inevitable losses ( $\mathrm{I}, 000 \mathrm{cc}$.) should be given with glucose. The practice of stimulating diuresis by giving infusions of electrolytes, such as sodium sulphate, can only cause a dangerous expansion of the extracellular fluid.

\section{Treatment}

The procedure outlined here applies to adults and excludes the special difficulties of parenteral therapy to infants. In every case the following questions must be examined separately:-

(I) How much water must be given?

(2) How much salt must be given?

(3) At what rate should the fluid be given?

(4) Should correction be made for disturbances of acid-base balance?

\section{How Much Water Must be Given?}

(a) Replace current losses. These are likely to be of the order of $2,500 \mathrm{cc}$. per day, allowing an inevitable loss of $1,000 \mathrm{cc}$. through the skin and lungs and a renal loss of $1,500 \mathrm{cc}$. To this must be added the volume of any abnormal loss of fluid from diarrhoea, vomiting, fistulae, etc.

(b) Estimate and replace deficit. To establish the quantity of water lost the following estimations may be made:-(I) Loss of body weight. Changes in the day to day estimation of body weight can be taken to reflect variations in body water. Therefore, neglecting the loss of weight due to an inadequate diet, a loss of $\mathrm{I} \mathrm{lb}$. body weight will be equivalent to a deficit of 500 cc. of water. (2) Packed cell volume, haemoglobin. In the absence of blood loss the red blood cells may be used to indicate variations in plasma volume. (3) Plasma protein level may similarly be used to detect haemoconcentration and the reduction in plasma volume. The change in the volume of each of these will be proportional to the diminution of circulatory. fluid, and may therefore be used to give an approximate estimation of the deficit thus:-

$$
\begin{gathered}
\frac{\mathrm{Hb}_{1}}{\mathrm{Hb}_{2}}=\frac{\text { Plasma } \operatorname{Pr}_{1}}{{\text { Plasma } \operatorname{Pr}_{2}}}=\frac{\text { Extracellular Fluid }}{\text { Normal Vol. (1 } 5 \mathrm{~L})_{\text {Normal Value }_{1}}} \\
\text { Pormal }
\end{gathered}
$$$$
\text { Abnormal } \text { Value }_{2}
$$

(4) The specific gravity of the plasma is easily measured ${ }^{13}$ and offers a ready method of determining water deficit. If the normal is taken as ? 1.027 then each 0.01 may be assessed as a deficit of 200 cc. of water.

The value of these tests is limited for it is necessary to assume an absolute figure of normality and neglect the range. They are further limited in longstanding cases and those with complications, particularly of bleeding, where alterations in red cells and plasma proteins occur simultaneously. No single investigation is reliable in assessing a patient's condition. In a particular case all possible evidence must be gathered together and interpreted against the background of thorough clinical examination. The estimation of the blood urea and alkali reserve are useful guides to the severity of the condition.

\section{How Much Salt Must be Given?}

In all cases of dehydration the most imfortant question to decide is whether the estimated water deficit must be replaced as normal saline or water alone. In practice this is extremely difficult because no direct measurement of the deficiency of sodium can be made. Estimation of serum sodium concentration cannot be used to indicate the quantity of sodium lost, and the chemical estimation takes too long to be of immediate use in assessing a case. $\AA$ deficit of sodium can be in- 
ferred when the renal excretion of chlorides is reduced. If a good volume of urine is passed an excretion rate below $3 \mathrm{gm}$. $/ \mathrm{l}$. (Fantus test) indicates a deficiency. However the limitations of this test must be realized. In Addison's disease and in renal failure excretion of chloride continues in spite of the deficiency. When sodium ions are lost in excess of chloride ions (e.g. in diarrhoea and in intestinal fistula) to maintain acid-base balance the kidney will excrete chloride ions in the presence of a plasma deficiency of sodium. Conversely, after surgical operations the ability of the kidney to excrete salt is greatly impaired, and a reduced urinary chloride does not indicate deficiency. ${ }^{5}$

Estimation of the amount of salt to be given must depend upon the losses indicated by the history and from clinical observation of signs and symptoms (Table I). The reduction of systolic blood pressure to a figure between 90 and 100 $\mathrm{mm}$. Hg. suggests a moderate loss of sodium, equivalent to 4 to 61 . of isotonic saline. If sodium deficiency is present, fluid replacement should be made initially with normal saline intravenously, and the amount required should be decided by the response of the patient. All urine passed should be collected and the choride content estimated. When this approaches 3 to $5 \mathrm{gm}$. $/ \mathrm{l}$. and the clinical condition has improved, isotonic saline should be replaced by half isotonic saline and glucose to make up the estimated deficit of fluid.

\section{Rate of Administration}

It is advisable to divide the day into eight hourly periods, before each of which clinical assessment of the patient's state is made and treatment ordered accordingly, a careful record of which is essential.

First period. Give one-third of the daily fluid requirement $(800 \mathrm{cc}$.) to which add one-half of the estimated deficit. In a moderately severe case, with a deficit of 61 ., the fluid prescribed for the initial period should be about 41 . Give 1 1. in the first hour and thereafter $500 \mathrm{cc}$./ hour, using normal saline in the presence of sodium deficiency, otherwise half normal saline and glucose.

Second period. Reassess the condition of the patient, taking particular note of the state of the circulation (venous P., B.P., pulse, respiration rate, urinary excretion (volume and chlorides), haemoconcentration (Plasma S.G., Hb. and P.C.V.). The disproportion between the deficit of salt and water should have been corrected during the first period. The volume of fluid to be given will consist of one-third of the daily requirement and one-half of the initial deficit of water. In all but the most severe cases give this orally with glucose or food, otherwise intravenously as glucose saline.

Third period. The clinical state of the patient $C$ should be assessed and if a deficit still exists the $\overrightarrow{\vec{F}}$ amount should be added to the normal require- $\frac{\text { ? }}{9}$ ment $(800 \mathrm{cc}$.) for that period.

\section{Correction for Acid-Base Balance}

Control of the reaction of the blood is achieved $\stackrel{\mathbb{D}}{\varrho}$ rapidly by the lungs and kidneys and significant कै disturbance either of acidosis or alkalosis cannot $\vec{\circ}$ occur unless such loss of water and electrolyte has occurred as to render the kidneys incapable of $\vec{\omega}$ correcting the abnormalities. Therefore it is of $\frac{\Omega}{8}$ the utmost importance to restore any salt and $\frac{0}{3}$ water lost. Isotonic saline should be given to cases of alkalosis or acidosis except those in the most $y$ severe acidosis, when one-sixth molar sodium $\stackrel{\omega}{-}$ lactate should be given at the ratio of $0.5 \mathrm{l}$. to every 15 vols. per cent. depression in the alkali reserve $\underset{+}{\omega}$ below 60 vols. per cent. The correction of severe 0 sodium deficiency by normal saline alone can? produce significant acidosis and therefore $I$. in $5 \mathrm{cs}$ of saline should be replaced by sodium lactate.

\section{Potassium Balance}

Recognition has only been given during the last few years to the fact that there are clinically in o portant conditions besides familial periods. paralysis and Addison's disease in which severe chronic disturbances of potassium distribution and balance may occur. Balance studies, supplemented in some instances by muscle analyses, performed on man and experimental animals, has revealed $a \stackrel{\mathbb{\perp}}{\stackrel{2}{\perp}}$ loss of cellular potassium in numerous conditions, $\overrightarrow{\overrightarrow{\hat{P}}}$ including diabetic coma, diarrhoea, vomiting, $\frac{0}{3}$ water deprivation, dietary potassium deficiency, alkalosis, shock, adreno-cortical tumours and nephritis.

A normal diet contains 2 to $4 \mathrm{~g}$. of potassium per diem, the ion being widely distributed, in $\frac{5}{3}$ concentrations of the order of 0.3 per cent. in animal and vegetable foods. Normally the kid- $O$ neys play the major part in the elimination of this ion, and are not effective in conserving it when the 음 body is deficient in potassium.

A typical concentration of potassium in intracellular water is $150 \mathrm{~m}$.Eq./1. (600 mg./100 cc.) N while the interstitial fluid contains a concentration close to that in plasma, about $5 \mathrm{~m} . \mathrm{Eq} . / \mathrm{l}$. N (20 $\mathrm{mg} . / \mathrm{roo} \mathrm{cc}$.). Most of the body potassium $\mathrm{\omega}$ (order of $15 \circ$ g.), therefore, resides in the tissues, about half is in muscle and only about $1 / 50$ in the extracellular fluid. Work with isotopic potassium has shown that there is an equilibrium between the intra- and extracellular potassium, but the precise $\frac{T}{\circ}$ mechanism by which potassium is maintained at $\frac{}{\mathbb{D}}$ 
itś relatively high concentration in cells remains to be elucidated. The Detection and Effects of Disturbances of
Potassium Distribution and Balance

A deficiency of potassium is here understood to involve such a depletion of the body content as obviously could not occur without loss of cell potassium.

If balance studies reveal a retention of potassium while the patient is under treatment, it may be concluded that a deficiency previously existed. Such studies are too elaborate and protracted for ordinary clinical practice and in any case only reveal a deficit in retrospect.

The published work has shown that a deficiency is reflected in loss of potassium from muscle, and in clinical research the analysis of a muscle biopsy has been used to provide evidence.

Potassium lost from the cells may be replaced to a variable degree by sodium..$^{14,15}$ The available evidence suggests that cell potassium loss alone does not produce symptoms, but replacement by sodium may do so. ${ }^{14,15}$ In clinical medicine the level of potassium in the extracellular fluid appears to be the most important factor in the production of symptoms and signs. Fluctuations in the extracellular potassium concentration are in most cases secondary to changes in the cell. In abnormal states the extracellular or serum level of potassium may be high, normal or low, depending on a combination of factors:-

(I) Movement of potassium in or out of cells. In a large number of conditions potassium has been shown to leave the cells for the extracellular fluid. Cellular dehydration is common to most of these conditions. The withdrawal of water, or the impaired nutrition of the cells appears to be responsible for failure to maintain the normal intracellular potassium concentration. However, dehydration is not the only factor concerned, since potassium also leaves the cells in anuria and acute nephritis. Potassium will move back into the cells when the abnormal condition affecting the cell is removed. This usually takes place early in the treatment of an acute illness, when the clinical signs of a reduction in potassium level must be expected.

A variety of natural and synthetic substances, including insulin and adrenaline, will produce acute effects on the distribution of potassium in the body, resulting in small reductions in the serum potassium concentration. Data in this field are fragmentary and the mechanisms by which the actions are exerted not understood.

(2) The state of renal function. If there is impairment of renal function any potassium lost from the cells may accumulate in the extracellular fluid and reach a toxic level. When renal function is normal, potassium liberated from cells is progressively excreted ; and alleviation of the causal condition will result in withdrawal of potassium by the cell from the extracellular fluid, in which it may then fall to a dangerous level.

(3) Dietary potassium. A normal meal contains about $2 \mathrm{~g}$. of potassium and when this amount is given as a single dose to a normal fasting person the serum potassium will rise approximately 0.75 m.Eq./l. (3 mg./100 cc.) within three hours. ${ }^{16}$ It is, however, generally agreed that large amounts of potassium can be given by mouth without toxic symptoms developing, provided kidney function is normal.

The intestinal fluid contains 5 m.Eq./l. (20 $\mathrm{mg}$./ $100 \mathrm{cc}$.) of potassium and therefore any chronic diarrhoea and vomiting will lead to a deficiency of potassium even in the absence of dehydration.

Rats on a potassium-deficient diet lose potassium from the muscle cell with partial replacement by sodium.

\section{Clinical Discussion}

Since it is the extracellular concentration of potassium that determines the onset of symptoms, it is important to know this level, and therefore methods of detecting its fluctuations are dis cussed. The flame photometer, necessary equip-? ment for the most rapid method of determination, is not yet widely available, and the chemical methods are time consuming. With large deviations from the normal serum potassium levels the E.C.G. shows characteristic changes and provides an indirect method for the detection of gross abnormalities. The method would seem to be specially advantageous for serial observations. ${ }^{17}$ At low levels the QT interval is prolonged, the RST segment depressed and the $T$ wave lowered, flattened and prolonged. At high levels the PR interval is prolonged, the $\mathbf{P}$ wave may disappear, the QRS complex is prolonged, the $T$ wave increases in amplitude and becomes peaked and eventually there is intraventricular block. Probably the single most important change in the E.C.G. with high levels of potassium is the alteration in amplitude of the $T$ wave with peaking. ${ }^{18}$ There is individual variation in the E.C.G. response to the serum potassium level and small departures from the normal are not detectable by this method.19,20 Early signs of a fall in serum potassium which may appear are an alteration of personality, consisting of irritability together with apathy. ${ }^{21}$ There may be asthenia and diminution of reflexes and tone. Eventually there may be extreme muscular weakness or virtual paralysis, 
bradycardia and shallow respiration which leads to cyanosis.

There is, at present, no easy way of detecting actual or incipient potassium deficiency. In clinical work interest is naturally focused on the extracellular concentration of potassium, and in the absence of a flame photometer the E.C.G. is valuable. In practice it is necessary to assume a potassium deficiency to exist when the necessary conditions for the production of the abnormality are present, and treatment should be instituted on the lines described in the concluding section.

\section{Diabetic Coma}

Atchley et al. ${ }^{22}$ demonstrated a loss of body potassium in the development of diabetic coma. They allowed two cases of diabetes to relapse into coma and estimated by balance studies the deficits of water, sodium, potassium and chloride. The loss of water and electrolytes increased as acidosis developed. In one case a typical daily negative potassium balance was $90 \mathrm{m.Eq}$. (3.5 g.) and a typical negative sodium balance was $50 \mathrm{~m} . \mathrm{Eq}$. (I.I g.). During recovery there was retention of water and electrolytes. They indicated that the initial polyuria is an important cause of electrolyte loss.

Danowski ${ }^{23}$ studied eight cases of diabetic coma and found that all had lost potassium. Oral administration of potassium salt led to retention of the cation in each case. In only one case was there a definite paralysis and even in this there was recovery with oral potassium.

There is a significant loss of potassium during the development of diabetic acidosis, and this loss will be facilitated if, during therapy, excess fluids are given ${ }^{24}$ or any substance that will promote diuresis.

\section{Conditions Involving Loss of Intestinal fuices}

Intestinal juices contain approximately the same concentration of potassium as the extracellular fluid and the large volume lost in diarrhoea, vomiting and intestinal fistulae will lead to a loss of potassium. This loss will be seriously aggravated by the concurrent dehydration.

This has been shown by Tobler, ${ }^{25}$ who analysed the muscles of babies who had died from diarrhoea and vomiting, and found a deficit of 30 per cent. in intracellular potassium. Darrow ${ }^{26}$ reported a deficit of 40 per cent. in potassium on a fat-free solid basis in the same circumstance. These findings are in accord with Darrow's ${ }^{26}$ balance studies. It is of interest to note that in the muscle composition study referred to above, Darrow found only in a few cases replacement of the lost potassium by sodium, such as occurs in experimental animals given excess DOCA or on a potassium-deficient diet.

In fatty diarrhoea, calcium is one of the many $\stackrel{2}{c}$ electrolytes lost. The loss of both potassium and $\vec{\Rightarrow}$ calcium ions may account for the relative rarity of $\stackrel{\vec{\rho}}{+}$ tetany in steatorrhoea in spite of a low blood cal- $\bar{c}$ cium in most cases. Engel ${ }^{27}$ has shown that in steatorrhoea, when the serum levels of both ions $\frac{\bar{c}}{\vec{D}}$ are low, the administration of potassium may $\stackrel{\mathbb{Q}}{\varrho}$ precipitate tetany.

\section{Renal Disease}

(a) Chronic nephritis. In this condition a $\overrightarrow{\vec{\omega}}$ negative potassium balance may result from the $\stackrel{\circ}{\circ}$ inability of the kidneys to conserve base.

(b) Acute nephritis and anuria. There is potassium loss from the cells and accumulation in the extracellular fluid which may lead to potassium $\omega$ intoxication. During the diuresis which accompanies recovery from anuria, there is a rapid excretion of potassium in addition to other electrolytes, and this is likely to produce a considerable loss of potassium.

\section{Haemorrhage, Burns, Shock}

In all these conditions potassium is lost from the cells. In burns the destruction of tissue wi contribute to the release of intracellular potassiug $\overrightarrow{0}$ and so add to the general loss of body potassium. In a severe case the coincident failure of reng function may lead to a potassium intoxication.

\section{Treatment}

If conditions which produced an intracellular potassium deficiency are allowed to continue, the cells will not take up potassium. Only by removing the underlying cause, for example, in dehydration by replacing fluid and electrolyte will potassium administration restore the normal intracellular concentration. The relatively low concentration of potassium normally present in extracellular fluid cannot be exceeded with safety, and therefore potassium should only be given when kidney function is normal so that if none is retained by the cells the cation can be excreted.

Oral administration is a therapeutically efficient method of administration ${ }^{23}$ and is safer than the intravenous or intramuscular method. If it is impracticable to feed a diet rich in potassium, for $\stackrel{N}{\sigma}$ example milk, fruit juices or meat broth enriched N with potassium chloride, it may be possible to administer a solution by stomach tube.

If oral administration is impracticable as, for example, in pyloric stenosis, one may have re- $\frac{D}{D}$ course to subcutaneous injection. Darrow recom- $\stackrel{\infty}{?}$ mends for this ' $\mathrm{K}$-lactate,' containing $4.0 \mathrm{~g}$. 궁 sodium chloride, $2.7 \mathrm{~g}$. potassium chloride and 
52 cc. of molar sodium lactate (15.3 g.) per litre. This solution should be administered slowly, $80 \mathrm{cc} / \mathrm{kg}$. over more than four hours, and preferably in eight hours. If other solutions of potassium are thought to be preferable, then the amount of potassium given may range from 30 to $150 \mathrm{~m}$. Eq. (1.2 to $6 \mathrm{~g} . / \mathrm{day}$ ) in an adult, and the maximum concentration of potassium in the solution should be $80 \mathrm{~m} . \mathrm{Eq} .(3.2 \mathrm{~g} . / 1) .{ }^{28}$ Intravenous injection of potassium salts carries considerable risk of potassium intoxication.

The amount of potassium given in cases of suspected potassium deficiency should be such that if none entered the cell the serum level would not be dangerously elevated; $0.25 \mathrm{~g}$. of potassium chloride per kg. body weight per diem, orally, is a safe dose. Such a dose will not result in potassium intoxication even if there is no deficiency.

There is as yet no generally approved method of treatment in cases of acute high serum potassium, but intravenous calcium and glucose ${ }^{29,30,31}$ have been used. It is possible that a combination of intravenous glucose and subcutaneous insulin might be more effective. ${ }^{32}$ Orally administered ion-exchange resins may prove to be of value in cases of longstanding elevation of serum potassium such as occurs in anuria, where the normal physiological mechanism for the elimination of potassium is inoperative.

\section{Conclusion}

In patients suffering from disturbances of water and electrolyte metabolism attention should be paid to the balance of both sodium and potassium ions, but laboratory tests remain inadequate to present a complete and unequivocal picture of a patient's condition; the fundamental changes are obscured as a result of the operation of corrective mechanisms. The immediate changes arising from disturbances of potassium metabolism are:-

(I) Accumulation of potassium in the extracellular fluid when renal function is reduced.

(2) Reduction of extracellular potassium when, on recovery, the cells take up potassium.

Methods of therapy consist of (a) replacement of water and such ions as are grossly deficient, thereby (b) facilitating the operation of the most important corrective mechanism, that provided by the kidneys. Where renal function is severely impaired special difficulties arise, notably in the treatment of a dangerously high level of serum potassium. The well developed technique of intravenous therapy, used when oral administration is impracticable, is hazardous when used for giving potassium salts.

The value of regulating sodium and water balances is established and to this may be addee the importance of attention to potassium metaf bolism.

\section{REFERENCES}

'VERNEY, E. B. (1946), Lancet, ii, 739, 781.

'BORST, J. G. G. (1948), Acta Med. Scand., Suppl. 207.

'MARRIOTT, H. L. (1946), Brit. med. F., i, 245, 285, 328.

'MCCANCE, R. A. (1936), Gouldstonian Lectures, Lancet, i, 643, $704,765,823$.

'WILKINSON, A. W.' BILLING, B. H., NAGY, G., and STEWART, C. P. (1949), Lancet, i, 640.

'MERRILL, A. J. (1946), Ұ. Clin. Invest., 25, 389.

'MERRILL, A. J., and CARGILL, W. H. (1947), f. Clin. Invest., 26, 1 I 90 .

'SCHEMM, F. R. (1942), Ann. Intern. Med., 17, 952.

'REID, H. A., and HUGHES, W. (1949), Lancet, i, 593.

${ }^{10}$ DOCK, W., and FRANK, N. R. (1950), Amer. Heart F., 40, 638.

"MUIRHEAD, E. E. HALEY, A. E. HABERMAN, S., and HILL, J. M. (1948), Blood, Special Issue No. 2, p. IoI.

${ }^{12 B U L L, ~ G . ~ M ., ~ J O E K E S, ~ A . ~ M ., ~ a n d ~ L O W E, ~ K . ~ G . ~(1949), ~}$ Lancet, ii, 220.

13VAN SLYKE, D. D., HILLER, A., PHILLIPS, R. A., HAMILTON, P. B., DOLE, V. P., ARCHIBALD, R. M., and EDER, H. A. (1950), ' Ұ. Biol.'Chem., 183, 331.

14HEPPEL, L. A. (1939), Am. F. Phys., 127, 385.

15FERREBEE, J. W., PARKER, D., CARNES, W. H., GERITY, M. K., A'TCHLEY, D. W., and LOEB, R. F. (I94I), Ibid., 135, 230.

${ }^{16}$ KEITH, N. M., and OSTERBERG, A. E. (1946), Proc. Staff Meeting Mayo Clinic, 21, 385 .
17HOWARD, J. E., and CAREY, R. A. (1949), 7. Clin. End., 9, 691. ${ }^{18}$ THOMSON, W. A. R. (1939), Lancet, i, 808.

19TARAIL, R. (1948), Am. F. Med., 5, 828.

"NADLER, C. S., BELLET, S., and LANNING, M. (1948), Ibid., 5,838 .

${ }^{2}$ HAWKINS, C. F., HARDY, T. L., and SAMPSON, H. H. O (195I), Lancet, 1. 318.

${ }^{22}$ ATCHLEY, D. W., LOEB, R. F., RICHARDS, JUN., D. W. BENEDICT, Ë. M., and DR̈ISCOLL, M. E. (I933), $\mathscr{f} . \vec{O}$ Clin. Invest., 12, 297.

${ }^{23}$ DANOWSKI, T. S., PETERS, J. H., RATHBUN, J. C., $\frac{\partial}{}$ QUASHNOCK, J. M., and GREENMAN, L. (1949), Ibid., 28, 1 .

${ }^{24 H O L L E R, ~ J . ~ W . ~(1946), ~ צ . A . M . A ., ~ I 31, ~} x 186$.

${ }^{25}$ TOBLER, L. (1910), Arch. f. exper. Path. u Pharmakol., 73, 566.

${ }^{26}$ DARROW, D. C. (1946), $\mathcal{~}$. of Paed., 28, 515.

${ }^{27}$ ENGEL, F. L., and MARTIN, S. P. (1948), 'Abstracts of Southern Society for Clinical Research,' $\mathrm{Am}$. ' F. Med., 4, 455 .

sELKINTON, J. R., and TARAIL, R. (1950), Ibid., 9, 200.

20FINCH, C. A., SAWYER, C. G., and FLYNN, J. M. (1946), D

Ibid., i, 337. 28, 54I.

${ }^{31}$ FENN, W. O. (1940), Phys. Rev., 20, 377.

32BYWATERS, E. G. (1944), F.A.M.A., 124, 1103. 